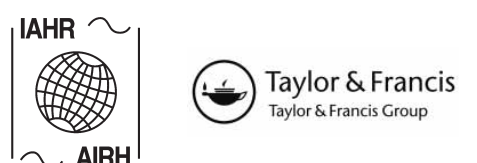

Journal of Hydraulic Research Vol. 49, No. 1 (2011), pp. 73-81

doi:10.1080/00221686.2010.534671

(C) 2011 International Association for Hydro-Environment Engineering and Research

Research paper

\title{
Water-surface oscillations in channels with axi-symmetric cavities
}

\author{
TOBIAS MEILE, Laboratory of Hydraulic Constructions (LCH), Ecole Polytechnique Fédérale de Lausanne \\ (EPFL), Lausanne, Switzerland. Now at: HydroCosmos SA, Vernayaz, Switzerland. \\ Email: tobias.meile@hydrocosmos.ch
}

JEAN-LOUIS BOILLAT, Laboratory of Hydraulic Constructions (LCH), Ecole Polytechnique Fédérale de Lausanne (EPFL), Lausanne, Switzerland.

Email: jean-louis.boillat@epfl.ch

ANTON J. SCHLEISS (IAHR Member), Laboratory of Hydraulic Constructions (LCH), Ecole Polytechnique

Fédérale de Lausanne (EPFL), Lausanne, Switzerland.

Email: anton.schleiss@epfl.ch (authorforcorrespondence)

\begin{abstract}
Transverse or longitudinal movements of a water body are observed for flows along cavities, river embayments, groyne fields or harbours. They are significant for certain flow conditions and geometrical properties. To study the effect of large-scale roughness on banks, 36 geometries of axi-symmetric, rectangular cavities were investigated in a laboratory flume under subcritical, turbulent free surface flow conditions. Significant movements of the water body were detected. The frequency of these periodic movements, identified by level and velocity observations, is in agreement with the natural frequency of the water body in a rectangular basin assuming the first-order mode of sloshing. Major movements of the water body, which lead to significant and periodic oscillations of the water surface, are avoided by excluding Strouhal numbers near 0.42 and 0.84 . For low aspect cavity ratios, the periodic water-surface oscillations are insignificant if the flow reattaches to the sidewalls of the widened channel reach.
\end{abstract}

Keywords: Cavities, embayment, open channel flow, water-surface oscillation, Strouhal number

\section{Introduction}

\subsection{Water-surface oscillations in cavities}

Oscillations of a water body can occur in groyne fields, river embayments, harbours and cavities. Research in this field primarily deals with free shear-layer oscillations, feedback mechanisms, resonance phenomena and passive or active open-loop control schemes (e.g. Rockwell and Naudascher 1978, Rowley and Williams 2006). As two of many examples, feedback mechanisms and free shear-layer oscillations occur by the impingement of vortices on a wall downstream of a sluice gate (Shuy and Chua 1999) or in free turbulent shear flows such as mixing layers (e.g. Chen and Jirka 1998). In river embayments, also called "Wando" in Japanese rivers (Muto et al. 2000, Nezu and Onitsuka 2002), horizontal coherent structures are generated by shear-layer instability between the main channel and the embayment. Studies on emerged groyne fields (Uijttewaal et al. 2001, Wirtz 2004, Weitbrecht 2004, McCoy et al. 2006) address mass exchange between the main channel and groyne fields, sedimentation of groyne fields, pollutant transport phenomena and navigation. In studies on harbour hydrodynamics (Wilson 1972, Sorensen 1978, van Rijn 1994, USACE 2002), the importance of harbour geometry and boundary conditions on the formation of unsuitable seiches in harbours is highlighted. The knowledge gained by the aforementioned research studies may be summarized as follows:

- The most important geometric parameter influencing the cavity flow pattern is the ratio between its lateral extent and length $\Delta B / L_{b}$, referred to as the cavity aspect-ratio.

- Flow in cavities, embayments or groyne fields is influenced by vortex shedding at the leading edge of the embayment.

- Flow recirculation in cavities, embayments or groyne fields can induce instabilities and lead to an instantaneous flow field different from the mean flow field.

Revision received 20 October 2010/Open for discussion until 31 August 2011.

ISSN 0022-1686 print/ISSN 1814-2079 online

http://www.informaworld.com 
- For free-surface flows (divergence-free flow), surface oscillations are observed for specific cavity geometries and particular flow conditions along the cavity (Naudascher 1963, Muto et al. 2000, Nezu and Onitsuka 2002, Wirtz 2004, Ohomoto et al. 2005).

This research addresses river embayments focusing on transverse oscillations to the main flow direction of a channel with cavities on both sides. These oscillations can hinder river navigation or reduce freeboard during floods.

\subsection{Investigations on oscillating cavity flow}

Rockwell and Naudascher (1978) classified self-sustaining, unstable and oscillating cavity flow into three groups: (i) fluiddynamic oscillations due to cavity shear-layer instability which are increased through a feedback mechanism, (ii) fluid-elastic oscillations for which an interaction of the fluid with the elastic displacement of a boundary occur, leading to the shear-layer instability and its amplification and (iii) fluid-resonant oscillations, where resonance conditions associated either with fluid compressibility or free-surface standing-wave phenomena are responsible for the shear-layer instability.

Fluid-resonant cavity oscillations can occur in open channels if the length of gravity waves is of the same order of magnitude than the cavity-characteristics length $L_{b}$ or the lateral extent $\Delta B$. If $\Delta B / L_{b}<0.5$, the cavity is shallow and longitudinal standing waves may be observed. If $\Delta B / L_{b}>1$, the cavity is deep and transverse standing waves may be present. Fluid-resonant oscillations of a deep cavity undergoing free surface flow are discussed by Naudascher (1963) for $\Delta B / L_{b}=2.0$. Fluidresonant oscillations are known to increase cavity-induced drag by $250 \%$ if the external flow is subsonic (McGregor and White 1970) which is always relevant in hydraulic engineering. Gharib and Roshko (1987) highlight the difference between the self-sustained oscillation mode, known as the low-drag regime, and the wake mode, known as the medium-drag regime. For the self-sustained oscillation mode, the cavity shear-layer stagnates at the downstream corner. For the wake mode, large-scale motion occurs across the cavity and mainstream boundary as well as, for two-dimensional (2D) flow with axi-symmetric cavities, across the channel axis. The flows studied by Gharib and Roshko (1987) oscillated in mediumdrag regime for cavity aspect ratios $\Delta B / L_{b}>0.77$.

The consideration of acoustic speed becomes important only at high flow velocities along cavities as the acoustic wavelength is of the same order of magnitude as the cavity length $L_{b}$. However, for nearly all oscillations in liquids, such as those investigated herein, $L_{b}$ is much smaller than the acoustic wavelength $\lambda$, and therefore $L_{b} / \lambda<<1$ such that the source and upstream perturbation propagation can be treated as purely hydrodynamic (Rockwell and Naudascher 1979).

In addition to the aspect ratio $\Delta B / L_{b}$, the upstream boundarylayer thickness relative to the cavity length and depth also influences the flow conditions inside the cavity (Rowley et al. 2002). If the upstream boundary-layer thickness decreases and/or Reynolds numbers increase, the flow is characterized by large-scale vortices shedding from the cavity leading edge (characteristic of wake mode). The flow becomes then periodic, with the fundamental frequency corresponding to the vortex shedding from the leading edge. The wake mode results from the feedback provided by the flow recirculation in the cavity and has the following main differences from the shear-layer mode (Rowley et al. 2002): The shear layer is not fixed to the downstream cavity edge, the mean streamlines above or along the cavities are deflected, the flow in the cavity has significantly higher levels of recirculation with a stagnation point occurring at the downstream cavity wall and intense oscillations, an order of magnitude larger than for the shear-layer mode, are observed.

Studies of jet-edge, cavity-edge and mixing-layer-edge oscillations indicated that the oscillation frequency can reasonably be predicted for certain geometries (Rockwell and Naudascher 1979). The dimensionless frequency of oscillations is expressed in terms of the Strouhal number $S=f \cdot L_{b} \cdot U^{-1}$, where $L_{b}$ is the cavity length, $U$ the mean velocity of the free stream and $f$ the frequency of the phenomenon.

The amplitude of cavity oscillations is generally more difficult to predict than their frequency. However, studies have found that peak excitations occur if oscillations of the shear layer are amplified due to cavity acoustic modes (East 1966, Erickson et al. 1986). Typical $S$ values resulting in peak excitations range between $S=0.3$ and 0.4 , or $S=0.6$ and 0.9 (Ziada 1994). For free surface flow conditions and deep cavities, peak excitations may also be observed at the resonant cavity frequency due to a transverse standing wave (Naudascher 1963). Finally, Rowley and Williams (2006) stated that cavity oscillations may not always be self-sustained, but slightly damped resonances, sustained by external disturbances.

\subsection{Studies on flow in groyne fields and embayments}

The flow patterns of embayments and groyne fields in emerged situations are similar since both produce shear-layer instabilities at the interface between the main stream and the dead zone. The aspect ratio $\Delta B / L_{b}$ of an embayment determines the shape and stability of the circulating flow and the stability itself has an effect on the mass and momentum exchange (Muto et al. 2000, Weitbrecht and Jirka 2001). In general, the mean flow in an emerged groyne field is dominated by a large single eddy if $0.5<\Delta B / L_{b}<1.5$. However, if $\Delta B / L_{b}<0.5$, a second eddy emerges in the upstream corner of the groyne field, whereas for $\Delta B / L_{b}>1.5$, the main eddy is split into two eddies in the transverse direction. For long distances between two groynes, flow reattachment can occur, leading to bank erosion and difficulties in navigation (Weitbrecht 2004). Even if the mean flow pattern in a groyne field is stable, the instantaneous flow field may vary significantly as the main and secondary eddies move in lateral and longitudinal directions leading to 
alternating mainstream flow velocities (Uijttewaal et al. 2001, Weitbrecht 2004) and the presence of a dynamic eddy (Yossef and de Vriend 2004).

Nezu and Onitsuka (2002) mentioned the importance of side-cavity open-channel flows. For flows along groyne fields, coherent horizontal structures are generated by shear-layer instabilities between the main channel and the open-channel side cavity. These structures lead to water-surface fluctuations inside the cavities and were measured in Japanese rivers using water-wave gauges (Chen and Ikeda 1996, Ikeda et al. 1999, Nezu and Onitsuka 2002). Kimura and Hosoda (1997) observed that the amplitude of water-surface fluctuations in an embayment increases with the Froude number. Nezu and Onitsuka (2002) confirm that the free surface in an embayment rises if the surface in the main channel falls and vice versa. Furthermore, they report that the period of the falling and rising water level is in relation with the seiche generated in semi-closed open-channel flows. Ohomoto et al. (2005) observed periods of water-surface oscillation in lab experiments on axi-symmetric groyne fields equal to the first-order mode of sloshing in a rectangular container. The same phenomenon was observed for prototype groyne fields on Elbe-River in Germany (Wirtz 2004).

Most studies on cavity flow features treat a single cavity or groyne fields on one channel side. The present study focuses on flow conditions and geometrical parameters leading to significant cavity oscillations for cavities on both sides of the main channel (Fig. 1). This goal is achieved by experimental tests of a large range of cavity geometries under subcritical, turbulent free surface flow. Experimental data were collected by studying systematically head losses generated by a succession of rectangular cavities on both channel banks (Meile 2007). The findings on flow conditions and geometrical parameters resulting in harmful oscillations can be used for example in groyne field and harbour design.

\section{Experimental setup}

\subsection{Test flume and geometrical configurations}

The test flume was $38.33 \mathrm{~m}$ long and had a mean slope of $1.14 \%$ (Fig. 1). From up- to downstream, the channel has the following sections: inlet reach $7.41 \mathrm{~m}$ long, a reach with rectangular cavities on side walls over $26.92 \mathrm{~m}$ and an outlet reach $4.0 \mathrm{~m}$ long. The sidewalls of the inlet reach are composed of wooden boards, while the rectangular cavity and the outlet reaches are formed by smooth limestone bricks. The channel bottom is of painted steel.

The channel base width was $B=0.485 \pm 0.002 \mathrm{~m}$. Three geometrical parameters, namely cavity length $L_{b}(=0.5,1.0$ or $2.0 \mathrm{~m})$, distance between two cavities $L_{c}(=0.5,1.0$ or $2.0 \mathrm{~m})$ and lateral cavity extent $\Delta B(=0.1,0.2,0.3$ or $0.4 \mathrm{~m})$ were systematically varied. The cavity aspect ratio defined as $A R=\Delta B /$ $L_{b}$ ranged from 0.05 to 0.80 , and the expansion ratio defined as $E R=(B+2 \Delta B) / B$ from 1.41 to 2.65 . The combination of three different values for $L_{b}$ and $L_{c}$ and four values of $\Delta B$ resulted in 36 different investigated geometrical configurations covering eight different aspect and four different expansion ratios.

\subsection{Instrumentation and measurements}

The test discharges were controlled by an electromagnetic flow meter. Flow conditions were investigated for up to 12 different steady discharges between $0.0037 \mathrm{~m}^{3} \mathrm{~s}^{-1}<Q<$ $0.1255 \mathrm{~m}^{3} \mathrm{~s}^{-1}$ for each of the 36 test configurations. The Froude number $\mathrm{F}=U(g h)^{-1 / 2}$ and the Reynolds number $\mathbf{R}$ $=U R_{h} v^{-1}$ were defined at cross-section 2-2 (Fig. 1). Characteristic values ranged between $0.37<\mathrm{F}<0.64$ and $6800<\mathrm{R}$ $<110,000$ for typical flow depths between $0.03 \mathrm{~m}<h<$ $0.34 \mathrm{~m}$ and mean flow velocities between $0.24 \mathrm{~m} \mathrm{~s}^{-1}<U<$ $0.80 \mathrm{~m} \mathrm{~s}^{-1}$. The water levels were recorded with ultra-sonic (US) sensors at a sampling rate of $16 \mathrm{~Hz}$ and a precision of $\pm 0.0005 \mathrm{~m}$ (Fig. 1). Some tests were repeated to perform velocity measurements by means of an ultrasonic velocity profiler (UVP). The $0.045 \mathrm{~m}$ long ultrasonic transducer, connected to the UVP, had an emitting frequency of $2 \mathrm{MHz}$ and an active diameter of $0.01 \mathrm{~m}$ (Fig. 2). The UVP was used to determine the streamwise and spanwise velocity components $u$ and $v$ in the widened channel reaches. Accurate velocity measurements were obtained by artificial flow seeding with hydrogen bubbles by application of direct current to two arrays of stainless steel wires immerged into the water (Meile et al. 2008a).

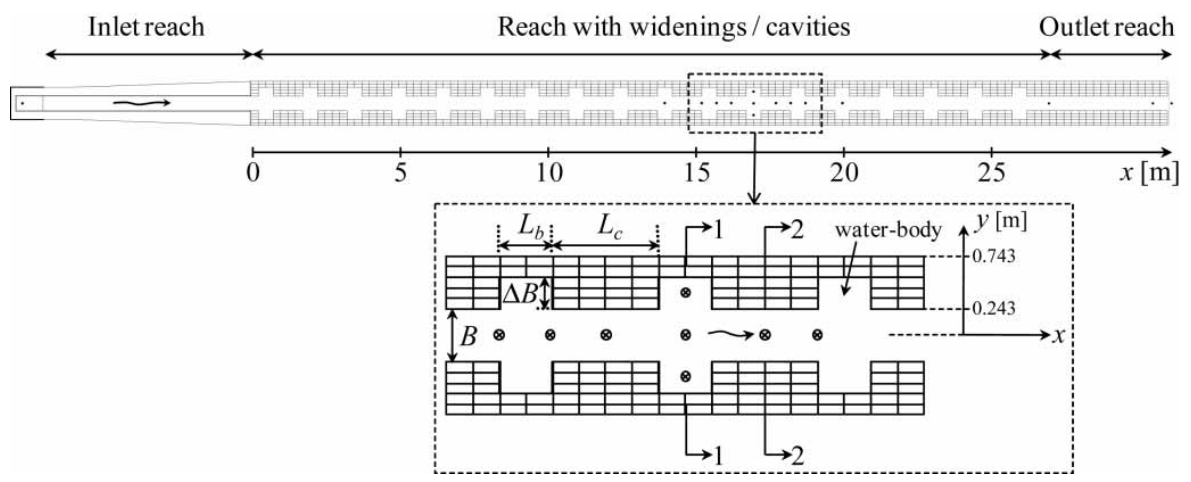

Figure 1 Overview of test flume and geometrical parameters of test configurations with US sensor locations $(\otimes)$ for level measurements 
Cross-section 1-1

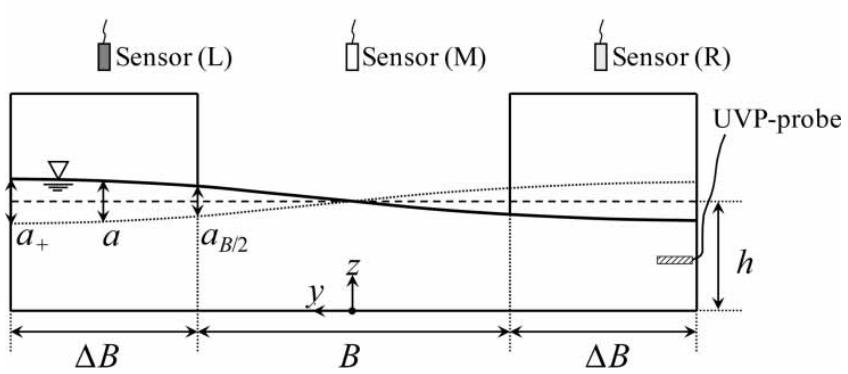

Figure 2 Cross-section 1-1 of Fig. 1 across oscillating water body in axi-symmetric cavities with US sensor locations for level measurements and UVP probe to measure spanwise velocity components

\section{Oscillations transverse to main flow direction}

\subsection{Amplitude and frequency of oscillating water-surface displacements}

The considered geometric parameters are defined in Fig. 2. The ultrasonic sensors (L), (M) and (R) are located at cross-section 11 in the left cavity, at the channel axis and in the right cavity. The mean water level $h$ is the time-averaged response from the $m$ water level measurements $h_{i}$. The mean wave height $a$ quantifies the water-surface oscillations around the mean water level $h$ and is calculated taking into account the standard deviation as

$$
a=2 \pi\left(\frac{1}{m} \sum_{i=m}^{m}\left(h_{i}-h\right)^{2}\right)^{1 / 2}
$$

The ultrasonic sensors measuring the oscillating movement of the water surface are located at $y= \pm(B / 2+\Delta B / 2)$. Assuming a sinusoidal water surface (Fig. 2), the mean wave height $a_{+}$at the widened channel side walls $y= \pm(B / 2$ $+\Delta B)$ becomes

$$
a_{+}=a\left[\sin \left(\frac{B / 2+B / 2}{B / 2+\Delta B} \frac{\pi}{2}\right)\right]^{-1}
$$

As the combined channel width and the widening $W=B+$ $2 \Delta B$ vary for the different test configurations, the mean wave heights $a$ or $a_{+}$cannot be directly compared. Assuming a sinusoidal water-surface profile, a representative mean wave height $a_{B / 2}$ at the junction between the main stream and cavities $y$ $= \pm B / 2$ is

$$
a_{B / 2}=a_{+} \sin \left(\frac{B / 2}{B / 2+\Delta B} \frac{\pi}{2}\right)
$$

The natural frequency or eigenfrequency $f^{n}$ of a water body in a rectangular basin assuming the $n$th sloshing mode (Fig. 2) is calculated as (e.g. Ohomoto et al. 2005)

$$
f^{n}=\frac{1}{2 \pi} \sqrt{\frac{n \pi g}{W} \tanh \left(\frac{n \pi h}{W}\right)}
$$

For low water level $h$ compared with the width $W$ of a semi-confined water body $h / W<<1, \tanh (n \pi h / W) \rightarrow n \pi h / W$, resulting in the seiche frequency (USACE 2002)

$$
f^{n}=\frac{n \sqrt{g h}}{2 W}
$$

Oscillating flow mechanisms are generally described with the Strouhal number S, based upon characteristic length and velocity, herein defined as

$$
\mathrm{S}=\frac{f^{n} L_{b}}{U}
$$

where $f^{n}$ is the natural frequency of an oscillating water body in a rectangular basin with $n$ nodes as defined by Eq. (4). Note that sloshing is influenced by the momentum transfer from the main flow into the cavity resulting in damping and excitation through the mixing layer as well as by the impingement of coherent structures at the downstream edge. Therefore, the Strouhal number is the important dimensionless parameter characterizing the excitation. In some cases, sloshing may be present, but too small to be detected as compared to other water-surface fluctuations.

\subsection{Energy required for maintaining water-surface oscillations}

An upper limit of energy dissipation due to periodic oscillations of the water body in the dominant mode ( $n=1$, Table 1$)$ is estimated based on: (1) potential energy $E_{\text {pot } 1}$ corresponding to the state of an incurved water surface is higher than potential energy $E_{\text {pot } 2}$ corresponding to the state of a horizontal water surface, (2) difference between the potential energies $\Delta E_{\text {pot } 1-2}$ if friction loss in the spanwise flow direction is neglected, provided by a one-time energy transfer into the system, (3) due to friction loss (damping), a portion of $\Delta E_{p o t 1-2}$ must be provided to the system at each oscillation by energy supplied by streamwise flow. For a semi-confined water body (Fig. 1) instead of a confined water body with streamwise velocity $=0$, the kinetic energy for the maximum inclined water surface would be zero. In this special case, the total energy for a single widened channel reach of length $L_{b}$ corresponds to the potential energy and is obtained by integration of the potential energy between $y=-(B / 2+\Delta B)$ and $y=+(B / 2+\Delta B)$ as (Meile 2007)

$$
E_{t o t, 1}=E_{p o t, 1}=\rho g L_{b}(B+2 \Delta B)\left(\frac{1}{2} h^{2}+\frac{1}{16} a_{+}^{2}\right)
$$

The total energy for the state corresponding to a horizontal water surface in the single widened channel reach is the sum of kinetic 
Table 1 Criteria to identify water-surface oscillations due to standing waves transverse to the main flow direction of mode $n=1$ or $n=2$

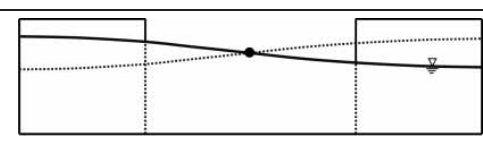

$n=1$ (standing wave with one node)

Criterion 1 based on phase difference $\Delta P_{\mathrm{L}-\mathrm{R}}$

If $170^{\circ}<\Delta P_{\mathrm{L}-\mathrm{R}}<190^{\circ}$

criterion 1 is satisfied

Criterion 2 based on relative wave heights $a_{r}=a / h$ at L, If $0.5 \cdot\left(a_{r \mathrm{~L}}+\mathrm{a}_{r \mathrm{R}}\right) / a_{r \mathrm{M}}>1.05$, $\mathrm{M}$ and $\mathrm{R}$

Criterion 3 based on Eq. (4) and the observed frequency criterion 2 is satisfied

If $\left(f_{o b s \mathrm{~L}}-f_{c a l}\right)$ or $\left(f_{\text {obs } \mathrm{R}}-f_{c a l}\right)<0.05$, criterion 3 is satisfied

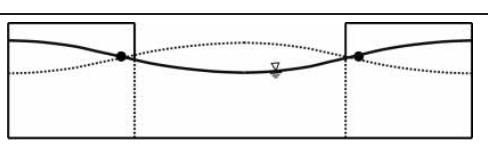

$n=2$ (Standing wave with two nodes)

If $\operatorname{abs}\left(\Delta P_{\mathrm{L}-\mathrm{R}}\right)<20^{\circ}$,

criterion 1 is satisfied

If $0.5 \cdot\left(a_{r \mathrm{~L}}+a_{r \mathrm{R}}\right) / a_{r \mathrm{M}}<0.95$, criterion 2 is satisfied

If $\left(f_{\text {obs } \mathrm{L}}-f_{c a l}\right)$ or $\left(f_{\text {obs } \mathrm{R}}-f_{c a l}\right)<0.1$, criterion 3 is satisfied

Note: L, left widening; $\mathrm{R}$, right widening; $\mathrm{M}$, channel axis.

and potential energies

$$
E_{t o t, 2}=\rho L_{b} h(B+2 \Delta B)\left(\frac{g h}{2}+\frac{\bar{V}_{t_{\max }}^{2}}{2}\right)
$$

where $\bar{V}_{t \text { max }}$ is the transverse velocity of a standing wave averaged in space between $-(B / 2+\Delta B)<y<+(B / 2+\Delta B)$ at the instant of horizontal water surface. Equation (8) is based on the assumption that horizontal velocities are significantly higher than vertical when the water-surface is horizontal. As a result, the kinetic energy is solely a function of the water body mass and the space-averaged transverse velocity.

Assuming full damping, the difference between the potential energy $\Delta E_{\text {pot } 1-2}$ at the two positions of the water surface (Fig. 2) gives the upper limit of energy dissipation due to transverse oscillations as

$$
\Delta E_{p o t, 1-2}=\rho g L_{b}(B+2 \Delta B)\left(\frac{1}{16} a_{+}^{2}\right)
$$

Alternatively, assuming no damping between the maximum inclined and horizontal states of the water surface, the maximum transverse velocity averaged in space $\bar{V}_{t \text { max }}$ is calculated using $E_{t o t, 1}=E_{t o t, 2}$

$$
\bar{V}_{t \text { max }}=\frac{a_{+}}{2} \sqrt{\frac{g}{2 h}}
$$

Since this velocity is space-averaged, it is lower than the maximum horizontal velocity in a standing wave $V_{t \text { max }}$ at the node (USACE 2002)

$$
V_{t \max }=\frac{a_{+}}{2} \sqrt{\frac{g}{h}}
$$

\subsection{Identification of standing waves spanwise to main flow}

The frequency of the observed oscillations transverse to the main flow direction corresponds to that of the most powerful free- surface oscillations measured by the US sensors. This frequency was identified by a fast Fourier transformation (FFT), as well as the phase $P$ of the water-surface oscillations at the three different US sensors of cross-section 1-1 (Fig. 2). The water-surface oscillations at the left (subscript L) and right (subscript R) side widenings are alternating for a phase difference $\Delta P_{\mathrm{L}-\mathrm{R}}$ approaching $180^{\circ}$ between the US sensors at the right and left embayments ( 1 node, $n=1$, Table 1 ). A phase difference $\Delta P_{\mathrm{L}-\mathrm{R}}$ tending to $0^{\circ}$ or $360^{\circ}$ would indicate that the water-surface oscillations at the left and right side widenings are produced simultaneously (two nodes, $n=2$; Table 1 ).

Three criteria as defined in Table 1 are used herein to determine whether observed water-surface oscillations are attributed to standing waves transverse to the main flow direction. These criteria are based on level measurements and FFT results; they include the mean wave heights at the right- and left-side cavities and along the channel axis, the phase difference between the signal at the right and left side cavities, and the frequency of the most powerful surface motion. It can be assumed that: (1) if all three criteria are satisfied for the first $(n=1)$ or second $(n=2)$ modes, the periodic water-surface oscillations of a semi-confined water body are definitely due to standing waves of the corresponding mode, (2) if two criteria are satisfied, periodic water-surface oscillations may be due to standing waves and (3) if one or no criterion is satisfied, water-surface oscillations are observed but neither periodical nor due to standing waves.

\section{Results and discussion}

\subsection{General flow characteristics}

Out of the total 429 tests, a standing wave with a single node according to Table 1 was definitely present in $29.6 \%$. In $7.5 \%$ of all tests, a standing wave of this mode might be possible. No periodic and significant water-surface oscillations were observed in the remaining $62.9 \%$. According to Table 1 , standing waves with two nodes were present in only $3.2 \%$ of the tests. In $11.4 \%$, a standing wave with two nodes might be possible. For 
the remaining $85.4 \%$, no periodic and significant water-surface oscillations with two nodes were observed. Tests involving a standing wave with two nodes were characterized by a cavity aspect ratio of $A R=0.40$ and an expansion ratio of $E R=$ 1.82. No significant longitudinal waves were observed in the experiments.

Alternate vortex shedding was often evidenced at the leading edges of the right- and left-side cavities. The coherent vortex structures are shed due to topographical forcing (Jirka 2001) and favoured by transverse flow oscillations. As they move downstream along the mixing layer between the main flow and the cavity, the coherent structures increase in size, the coherent structures increase in size. Depending on the flow velocity, the frequency and the relative amplitude of the transverse oscillations, the vortices are trapped into the cavity or entrained by the main flow. In long cavities, coherent structures may collapse before arriving at the downstream cavity edge.

Two different cavity-flow types were observed, namely flow recirculation and flow reattachment to side walls of the cavities. The flow recirculation can be divided again into a squaregrooved flow type for which $L_{b} \cong \Delta B$ and a normal recirculating flow type with an elongated main and a smaller secondary eddy according Uijttewaal et al. (2001) and Weitbrecht (2004). A simplified criterion for flow reattachment is $\Delta B / L_{b}<0.10$ (Meile et al. 2008b). With regard to the cavity aspect ratio, it can be concluded that the probability of transverse oscillations (single-node mode) increases with the cavity aspect ratio $\Delta B / L_{b}$ (Fig. 3 ). It is also evident that oscillations are not present for reattachment flow conditions.

Around aspect ratios of 0.3 and 0.4 , a fall and subsequent rise in the curves in Fig. 3 is observed, which reduces as the tests with "possible oscillations" are attributed to non-oscillating for $\Delta B$ / $L_{b} \leq 0.3$ and to definitely oscillating tests for $\Delta B / L_{b} \geq 0.4$. Further, the fall and rise of the curves reveal that the phenomenon of transverse oscillations is not only governed by the cavity aspect ratio, because the Strouhal number also has an effect (Section 4.3).

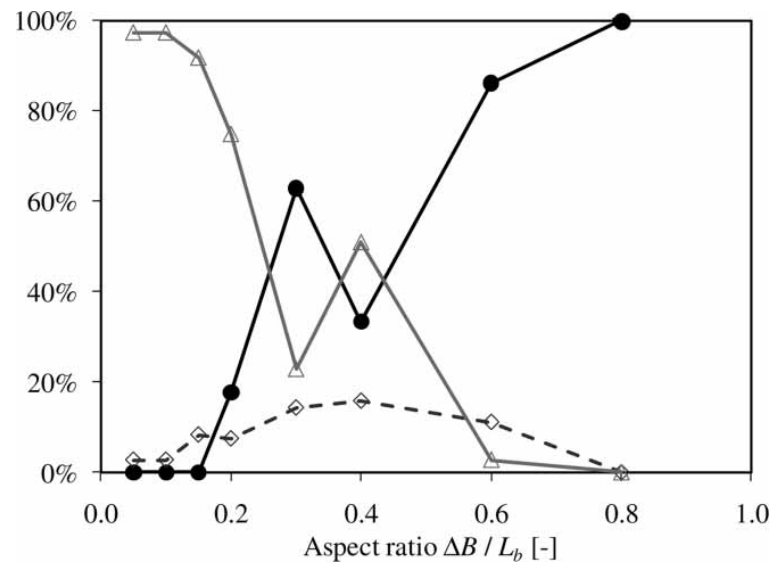

Figure 3 Classification of tests according to criteria of Table 1 into flows with $(\bullet)$ definitely present, $(\diamond)$ possible or $(\Delta)$ no oscillations

\subsection{Frequency of cavity oscillations}

The observed and calculated frequencies $f_{\text {obs }}$ and $f_{\text {cal }}$ of standing waves at the dominant single-node mode at cross-section 1-1 are compared for all tests with definite oscillations (Fig. 4). According to Eq. (4), higher frequencies correspond to higher water levels for a given geometry and mode. These tests lie in the transition regime for which the assumption of relative flow depth $h$ / $W<<1$ is no longer valid. The simplified Eq. (5) deviates from the exact expression for higher frequencies.

Regularly shedding vortices are assumed to be present in all tests but were clearly identified at the leading cavity edge using dye to detect important oscillations spanwise to the main flow. The observed vortex shedding frequency $f_{\text {shed }}$ agreed well with the calculated frequency $f_{\text {cal }}$ from Eq. (4), as well as the observed frequency $f_{\text {obs }}$ of the water-surface oscillations, identified by the FFT analysis (Meile 2007).

It may be concluded that vortex shedding and water-surface oscillations are coupled phenomena. The frequency of oscillations in axi-symmetric cavities transverse to the main flow direction is reasonably predicted with Eq. (4). Equation (5) is less adequate for the transition regime with higher relative flow depths, resulting in overestimations of 2.0 and $7.8 \%$ for $h / W$ $=0.11$ and 0.22 , respectively.

\subsection{Amplitude of cavity oscillations}

The relative height of water-surface oscillations is related to the Strouhal number, obtained by $f_{c a l}$ from Eq. (4). Significant peaking values of $\left(a_{B / 2} / h\right)^{2}$ occur for Strouhal numbers $S \cong$ 0.42 and $S \cong 0.84$ (Fig. 5). A third value leading to the peak values of the water-surface oscillations likely exists at $\mathrm{S}=$ 1.26. The mean wave heights at the side walls $a_{+}$reach values

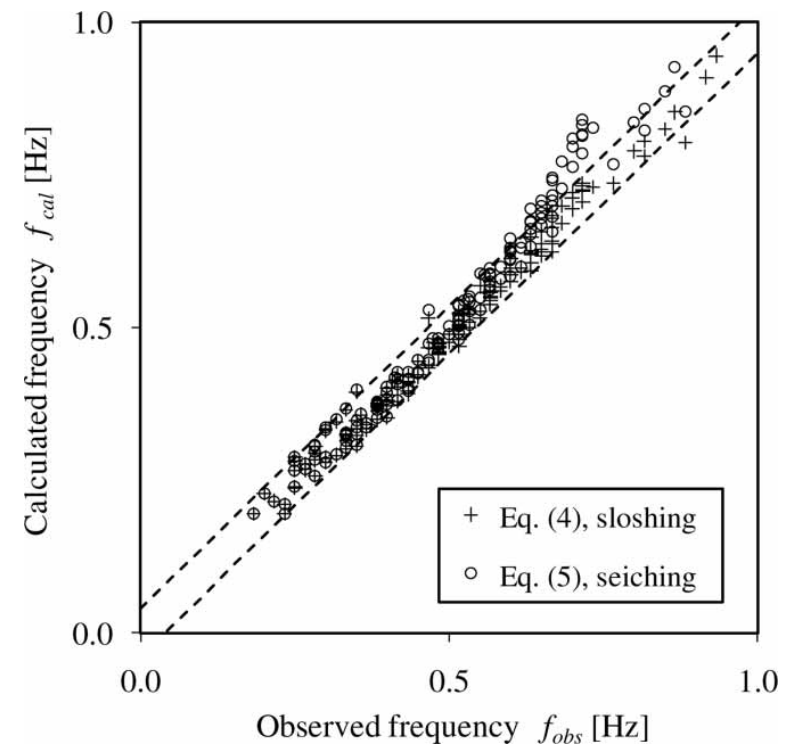

Figure 4 Comparison of calculated frequencies $f_{\text {cal }}$ by Eqs. (4) and (5) with observed frequencies $f_{\text {obs }}$ for all tests with definite water body oscillations ( $n=1$; all three criteria of Table 1 satisfied) 


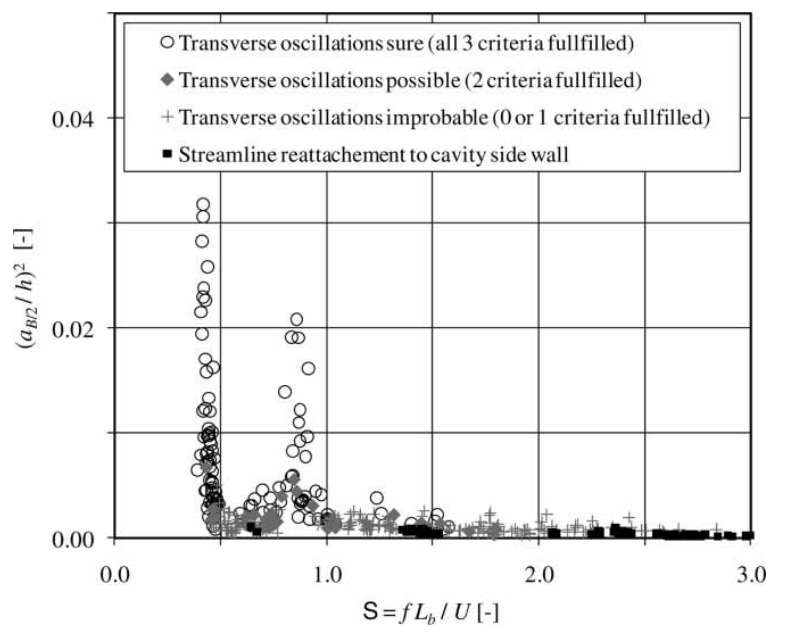

Figure 5 Square value of relative wave height at the line separating cavity from the mainstream $(y= \pm B / 2)$ as a function of Strouhal number S

up to $30 \%$ ( $\mathrm{S}=0.42)$ and $22 \%(\mathrm{~S}=0.84)$ of the mean flow depth $h$, respectively. At a distance $y= \pm B / 2$, for which mean wave heights $a_{B / 2}$ can be compared in all tested geometrical configurations, the values still reach $18 \%(S=0.42)$ and $15 \%(S=0.84)$ of the mean flow depth.

The amplification of the transverse oscillations at $S=0.42$, 0.84 and 1.26 is due to the impingement of downstream travelling coherent structures at the downstream cavity edge (e.g. Rowley et al. 2002) and can be explained physically as follows. Assume $T_{1}=L_{b} \cdot U^{-1}$ as time needed by a vortex to travel from the leading cavity edge to the cavity end and $T_{2}$ $=f^{-1}$ as time between the shedding of two vortices at the same edge. If $T_{1}$ corresponds to a multiple of $0.5 T_{2}$, the coherent structure travelling downstream interferes with the downstream cavity edge. Then, $\mathrm{S}=f L_{b} U^{-1}$ is a multiple of 0.5 . The experimentally determined Strouhal numbers of $0.42,0.84$ and 1.26 are of course lower than $0.5,1.0$ and 1.5 since the flow velocities along the channel side walls and the mixing layer are less than the mean channel flow velocity.

The experiments with flow reattachment to the side walls of the widening have mean relative wave heights lower than $3 \%$. For these tests, transverse movements of the water body resulting from a standing wave are negligible since the reattaching streamlines stabilize the flow pattern. The formation of large-scale recirculation eddies inside the cavities is suppressed, and thus the hydrodynamic feedback mechanism leading to the wake mode and the amplification of the standing wave is absent (Gharib and Roshko 1987, Rowley et al. 2002).

Axi-symmetric configurations, as herein tested, clearly trigger water-surface oscillations. Remarkable oscillations of up to $12 \%$ of the mean flow depth, spanwise to the main flow direction, were also observed by Ohomoto et al. (2005) for axi-symmetric groynes with an aspect-ratio $\Delta B / L_{b}=0.5$ and an expansion ratio of $(B+2 \Delta B) / B=2$. The observed periods of water-surface oscillation were equal to the first-order mode of sloshing in a rectangular container. The water-surface oscillations have been attributed to the interactions of large-scale eddies in the groyne fields along both channel sides and were likely caused by separation eddies behind the first groyne (Ohomoto et al. 2005). In the present tests with comparable geometry and mean flow depth, the water-surface oscillations are of the same order of magnitude as $a_{+} / h=14$ to $17 \%$.

\subsection{Transverse velocity components}

Measurements of transverse velocity components confirm that the periodic oscillations of the water surface are due to periodic, transverse oscillations of the water body contained in the widened channel reach (standing waves). The main frequency of the watersurface oscillations $\left(f_{o b s}=0.47 \mathrm{~Hz}\right)$ is close to the eigenfrequency of the water body $\left(f_{\text {cal }}=0.44 \mathrm{~Hz}\right)$ and corresponds exactly to the frequency of the cycle observed in the transverse velocity components $(f=0.47 \mathrm{~Hz})$. The cyclic behaviour of the transverse velocities near the channel axis (Fig. 6a) as well as the cyclic behaviour of the space-averaged transverse velocity (Fig. 6b) can be clearly observed.

(a)
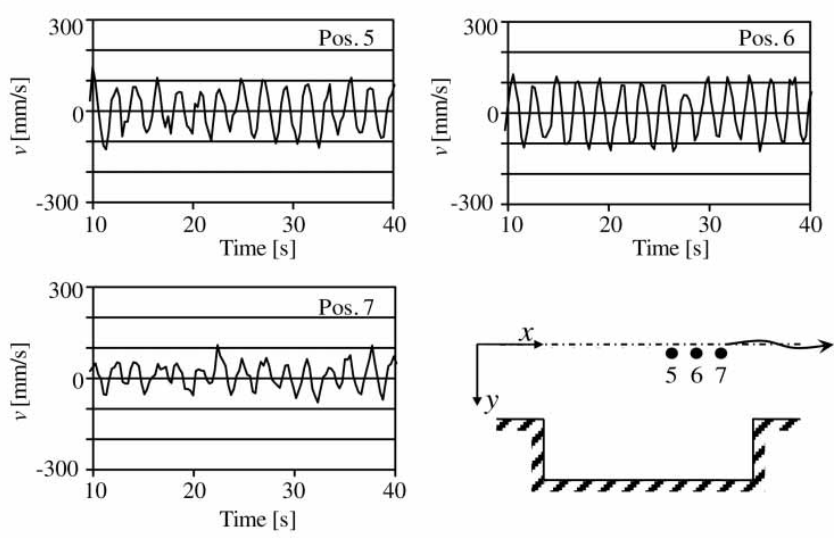

(b)
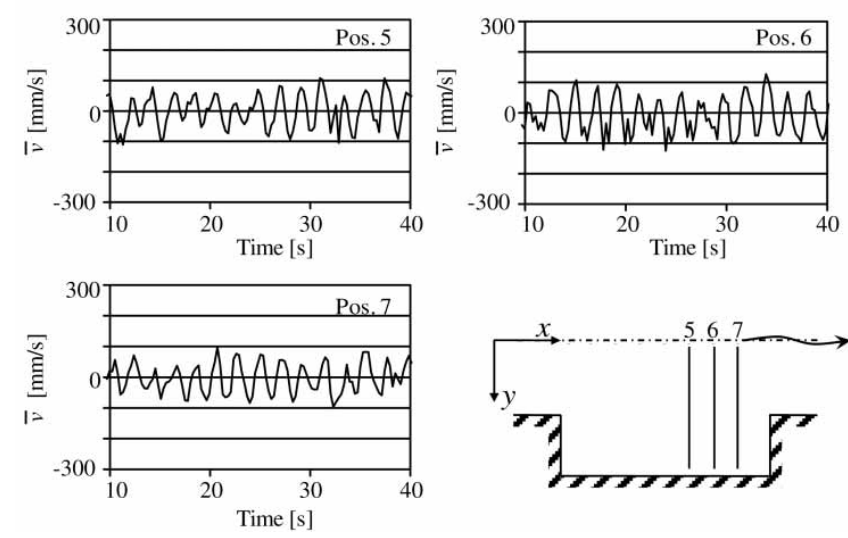

Figure 6 Transverse velocity components at various locations $x$ for $L_{b}$ $=1.0 \mathrm{~m}, L_{c}=1.0 \mathrm{~m}$ and $\Delta B=0.4 \mathrm{~m}\left(Q=0.036 \mathrm{~m}^{3} \mathrm{~s}^{-1} ; h=\right.$ $0.132 \mathrm{~m}$ ). Vertical position of velocity measurement at $z=0.5 \mathrm{~h}$. (a) Local transverse velocity near channel axis and (b) space-averaged transverse velocity $(0.05 \mathrm{~m}<y<0.56 \mathrm{~m})$ 
The observed maximum transverse velocities are of the same order of magnitude as the maximum calculated transverse velocity $V_{t \text { max }}=0.096 \mathrm{~m} \mathrm{~s}^{-1}$ using Eq. (11). The maximum observed, space-averaged velocity seems to be somewhat higher than the calculated space-averaged velocity $\bar{V}_{t} \max$ $=0.068 \mathrm{~m} \mathrm{~s}^{-1}$ according to Eq. (10). Two possible reasons may explain this discrepancy: vertical position of measurement beam and/or lack of measurements close to side wall of cavity.

\section{Conclusions}

This study covers the behaviour of movements of a water body perpendicular to the main flow direction. For many of the investigated configurations in a long channel equipped with a large number of axi-symmetric rectangular side cavities, significant water-surface oscillations were observed. These were analysed for 36 different geometrical configurations with aspect ratios ranging from 0.05 to 0.80 and expansion ratios ranging from 1.41 to 2.65 . Up to 12 different discharges were investigated for each configuration, leading to various flow conditions. The results should be applied to axi-symmetric cavity configurations with the above stated aspect and expansion ratios and subcritical flow conditions.

The water-surface oscillations are periodic and significant for geometrical configurations with flow recirculation in the embayments. The periodic oscillations of the water surface (standing waves) result from movements of the water body contained inside the widened channel reach transverse to the main flow direction. Several factors favour important oscillations of the water body: axi-symmetric and regularly arranged cavities, high expansion ratios and relatively high aspect ratios as well as impingement of coherent structures at the downstream cavity edge. Impingement occurs for Strouhal numbers close to 0.42 and 0.84 .

If no periodic oscillations are observed, the relative mean wave height at the lateral side walls of the widened channel reach is less than $3 \%$. If strong water-surface oscillations occur due to standing waves, the mean wave height can reach up to $30 \%$ of the mean flow depth. The transverse velocity components, measured near the channel axis, demonstrate similar periodic behaviour as the water-surface oscillation, confirming that the semi-confined water body is seiching. The observed frequencies of the watersurface oscillations match the eigenfrequencies of the water body formed by the widened reach. This frequency can be predicted by the sloshing theory in a rectangular basin.

The amplitude of water-surface oscillations is more difficult to predict than the frequency. However, for flows along rectangular cavities and embayments, the experiments clearly highlighted critical values of the Strouhal number near 0.42 and 0.84 where peak excitations of the water-surface oscillations occur. For practical applications which require a reduction of water-surface oscillations and transverse flow movements such as harbour, groyne field or embayment design, critical Strouhal numbers should be avoided. For low aspect ratios, if the flow reattaches the side walls of the widened channel reach, periodic watersurface oscillations are insignificant.

\section{Acknowledgements}

This research was funded by the Swiss Federal Office for the Environment (FOEN). The measurement equipment for velocity measurements was supported by Met-Flow SA, Lausanne, Switzerland.

\section{Notation}

$a$

$a_{B / 2}$

$a_{+}$

$A R$

$B$

$\Delta B$

E

ER

$f$

F

$g$

$h$

$h_{i}$

$L_{b}$

$L_{c}$

$n$

$\mathrm{R}$

$\mathrm{S}$

$U$

$\bar{V}_{t \max }$

$V_{t \max }$

$W$

$(u, v, w)$

$(x, y, z)$

$=$ mean wave height at ultrasonic sensor

$=$ mean wave height at $y= \pm B / 2$

$=$ mean wave height at $y= \pm(B / 2+\Delta B)$

$=$ aspect ratio of the cavity $=\Delta B / L_{b}$

$=$ channel base width at cross section 2-2

$=$ lateral cavity extent

$=$ energy

$=$ expansion ratio of the cavity $=(B+2 \Delta B) / B$

$=$ frequency of oscillations

$=$ Froude number

$=$ gravitational acceleration

$=$ mean flow depth

$=$ instantaneous flow depth

$=$ cavity length

$=$ distance between two cavities

$=$ number of nodes of a standing wave

$=$ Reynolds number

$=$ Strouhal number

$=$ mean flow velocity in cross-section $2-2$

$=$ space-averaged maximum transverse velocity of a standing wave

$=$ maximum transverse velocity of a standing wave

$=$ width of semi-confined water body

$=$ streamwise, spanwise and vertical velocity components

$=$ axis of Cartesian reference system

\section{Subscripts}

obs, cal = observed and calculated

shed $\quad=$ frequency at which vortex shedding was observed visually

$\mathrm{L}, \mathrm{M}, \mathrm{R} \quad=$ left, middle and right

\section{References}

Chen, F., Ikeda, S. (1996). Experimental study on horizontal separation eddies in open-channel flow with groins. Annual J. Hydraulic Engng. JSCE 40, 787-792.

Chen, D., Jirka, G.H. (1998). Linear instability analyses of turbulent mixing layers and jets in shallow water layers. J. Hydraulic Res. 36(5), 815-830.

East, L.F. (1966). Aerodynamically induced resonance in rectangular cavities. J Sound and Vibrations 3(3), 277-287. 
Erickson, D.D., Durgin, W.W., Maguire, C.F., Moeller, M.J. (1986). Shear layer coupling with side-branch resonators. Forum on Unsteady Flow, ASME Publication No. FED39, 43-45.

Gharib, M., Roshko, A. (1987). The effect of flow oscillations on cavity drag. J. Fluid Mech. 177, 501-530.

Ikeda, S., Yoshike, T., Sugimoto, T. (1999). Experimental study on the structure of open channel flow with impermeable spur dikes. Annual J. Hydraulic Engng. JSCE 43, $281-286$.

Jirka, G.H. (2001). Large scale flow structures and mixing processes in shallow flows. J Hydraulic Res. 39(6), 567-573.

Kimura, I., Hosoda, T. (1997). Fundamental properties of flows in open channels with dead zone. J. Hydraulic Eng. 123(2), 98-107.

McCoy, A., Constantinescu, G., Weber, L. (2006). Exchange processes in a channel with two vertical emerged obstructions. Flow, Turbulence and Combustion 77(1-4), 97-126.

McGregor, D.W., White, R.A. (1970). Drag of rectangular cavities in supersonic and transonic flow including the effects of cavity resonance. J. AIAA 8(11), 1959-1964.

Meile, T. (2007). Influence of macro-roughness of walls on steady and unsteady flow in a channel. PhD Thesis 3952 Ecole Polytechnique Fédérale de Lausanne, and Communication 36, Laboratory of Hydraulic Constructions, A.J. Schleiss, ed., EPFL Lausanne, Switzerland.

Meile, T., De Cesare, G., Blanckaert, K., Schleiss, A.J. (2008a). Improvement of Acoustic Doppler Velocimetry in steady and unsteady turbulent open-channel flows by means of seeding with hydrogen bubbles. Flow Measurement and Instrumentation 19(3-4), 215-221.

Meile, T., Boillat, J.-L., Schleiss, A.J. (2008b). Dämpfende Wirkung von grossmassstäblichen Uferrauheiten auf Schwall- und Sunkerscheinungen in Flüssen (Damping effect of large scale embankment roughness on hydro peaking in rivers). Wasserwirtschaft 98(12), 18-24 [in German].

Muto, Y., Imamoto, H., Ishigaki, T. (2000). Turbulence characteristics of a shear flow in an embayment attached to a straight open channel $4^{\text {th }}$ Intl. Conf. Hydroscience and Engineering, Seoul, Korea, 232-241.

Naudascher, E. (1963). On the role of eddies in flow-induced vibrations. $10^{\text {th }}$ IAHR Congress, London UK 3, 61-72.

Nezu, I., Onitsuka, K. (2002). PIV measurements of side-cavity open-channel flows: Wando model in rivers. J. Visualization 5(1), 77-84.

Ohomoto, T., Nirakawa, R., Watanabe, K. (2005). Interaction between water surface oscillations and large eddies in an open channel with spur dikes. $31^{\text {st }}$ IAHR Congress, Seoul, Korea Abstract 1, 347-348 (CD-Rom).

Rockwell, D., Naudascher, E. (1978). Review: Self-sustaining oscillations of flow past cavities. J. Fluids Engng. 100(6), 152-165.

Rockwell, D., Naudascher, E. (1979). Self-sustained oscillations of impinging free shear layers. Annual Review Fluid Mech. 11, 67-94.

Rowley, C.W., Colonius, T., Basu, A.J. (2002). On self-sustained oscillations in two-dimensional compressible flow over rectangular cavities. J. Fluid Mech. 455, 315-346.

Rowley, C.W., Williams, D.R. (2006). Dynamics and control of high-Reynolds-number flow over open cavities. Annual Review Fluid Mech. 38, 251-276.

Shuy, E.B., Chua, H.C. (1999). Fluid-dynamic feed-back in shear layer oscillation below a submerged sluice gate. $J$. Hydraulic Res. 37(11), 107-120.

Sorensen, R.M. (1978). Basic coastal engineering. Wiley, Brisbane.

Uijttewaal, W.S.J., Lehmann, D., van Mazijk, A. (2001). Exchange processes between a river and its groyne fields: Model experiments. J. Hydraulic Eng. 127(11), 928-936.

USACE (2002). Engineering and design, coastal engineering manual, harbour hydrodynamics. Report EM 1110-2-1100. Department of the Army, US Army Corps of Engineers, Washington DC.

van Rijn, L.C. (1994). Principles of fluid flow and surface waves in rivers, estuaries, seas, and oceans. Aqua Publications, Amsterdam NL.

Weitbrecht, V. (2004). Influence of dead-water zones on the dispersive mass transport in rivers. Dissertation 2004/1. Institut für Hydromechanik, Universität Karlsruhe, Karlsruhe, Germany.

Weitbrecht, V., Jirka, G.H. (2001). Flow patterns and exchange processes in dead zones of rivers. Intl. Symp. Environmental Hydraulics ISEH and IAHR Phoenix AZ, 1-6.

Wilson, B.W. (1972). Seiches. Advances in hydroscience. Academic Press, New York NY, 1-94.

Wirtz, C. (2004). Hydromorphologische und morphodynamische Analyse von Buhnenfeldern der unteren Mittelelbe im Hinblick auf eine ökologische Gewässerunterhaltung. Ph.D. Thesis. Freie Universität Berlin, Berlin [in German].

Yossef, M.F.M., de Vriend, H.J. (2004). Mobile-bed experiments on the exchange of sediment between main channel and groyne fields. Proc. River Flow 2004 Naples 1, 127-133.

Ziada, S. (1994). A flow visualization study of flow-acoustic coupling at the mouth of a resonant side-branch. J. Fluids and Structures 8(4), 391-416. 